\title{
Numerical Analysis of the Rapid Solidification Process of NiTi Binary Alloy
}

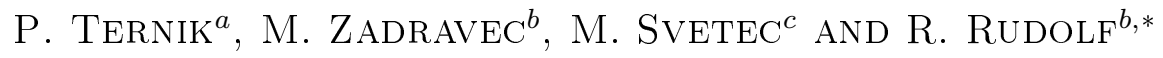 \\ ${ }^{a}$ Ternik Primož — Private Researcher, Bresterniška 163, Bresternica, Slovenia \\ ${ }^{b}$ Faculty of Mechanical Engineering, Smetanova 17, Maribor, Slovenia \\ ${ }^{c}$ Pomurska akademsko znanstvena unija, Lendavska 5a, Murska Sobota, Slovenia \\ (Received September 4, 2014; in final form November 12, 2014)
}

\begin{abstract}
The objective of the present work is to analyse the solidification process of NiTi binary alloy. The physical medium is taken as an incompressible fluid where the heat is transferred by conduction and convection, including the thermal phase change phenomenon. The last one is modelled by the improvement procedure called enthalpyporosity formulation, where the liquid-solid mushy zone is treated as a porous zone with porosity equal to the liquid fraction. The numerical model is based on the finite volume method in body fitted coordinates with a SIMPLER scheme to couple the pressure and velocity fields. The results are presented for the velocity and temperature field, as well as for the NiTi mass fraction during the solidification process.
\end{abstract}

DOI: $10.12693 /$ APhysPolA.128.43

PACS: 64.70.Ja, 81.30.Fb, 44.05.+e

\section{Introduction}

$\mathrm{Ni}-\mathrm{Ti}$ based alloys are the most important practical shape memory alloys (SMA) with excellent mechanical properties, good corrosion resistance and excellent biocompatibility. Moreover, in these alloys there are many phase transformations, which include diffusionless martensitic transformations, shape memory and superelastic effects and also diffusional transformations. These materials belong to a group of shape memory materials which are among the group known as functional materials.

The first observation of the shape memory behaviour of materials goes back to 1932, when the Swedish researcher Olander first noticed this phenomenon on a sample of gold and cadmium (Au-Cd). In 1962, Buehler and his colleagues in the Naval Ordnance Laboratory observed the shape memory effect in the alloy of nickel and titanium. This alloy was named NiTinol (Nickel-Titanium Naval Ordnance Laboratory) [1].

The use of NiTinol wire in orthodontics was introduced by Andreasen in 1971 and is now used increasingly because of its unique elastic properties [2]. The main advantage of NiTi orthodontic wires compared to conventional (e.g. stainless steel) wires is their ability to deliver constant stress to push the teeth into the proper position. This property makes this product much more efficient, thus reducing the length of orthodontic treatment for patients. The level of stress in the wire can be controlled through its chemical composition and its thermomechanical history. It can also control the geometrical dimensions, as is the case with conventional wires [3].

\footnotetext{
* corresponding author; e-mail: rebeka.rudolf@um.si
}

The understanding of the phenomenon of martensitic transformation did not develop rapidly. This is because the Ni-Ti alloy system [4] is quite a complicated system (see Fig. 1). Various precipitates, which formatted during casting or under certain heat-treatments, had not been understood well from the proposed phase diagram.

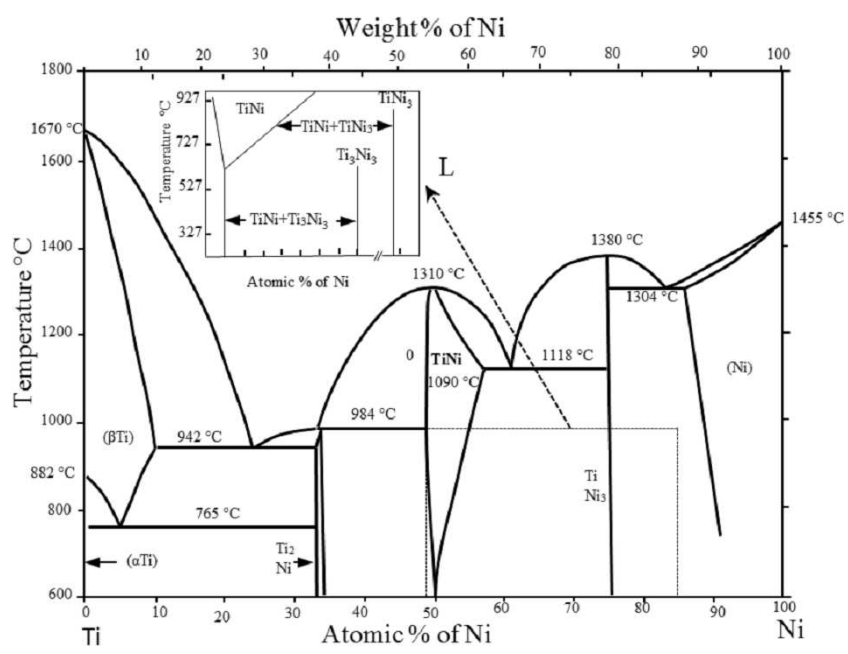

Fig. 1. Ni-Ti phase diagram.

NiTi materials show two special behaviours: shape memory effect (SME) and superelastic effect (SE) (Fig. 2) [5]. Both effects are characterised by martensitic phase transformation, which is caused by changing the temperature (shape memory) or application of stress (superelastic effect) leading to changes of phase or microstructure. The austenite phase is stable at high temperatures and small stresses, and the martensitic phase is stable at low temperatures and high stresses. These phases have different crystal structures. The austenitic phase has a body-centred cubic crystal structure, while the martensitic phase has a monoclinic crystal structure. 
The transformation from one structure to the other does not occur by diffusion of atoms. The transformation is caused by shear lattice distortion. The martensitic transformation is known as diffusionless transformation. SME allows the alloy to return to its previous shape when it is heated above the $A_{\mathrm{f}}$ temperature. The SMA regains its original shape by transforming into the austenite phase.

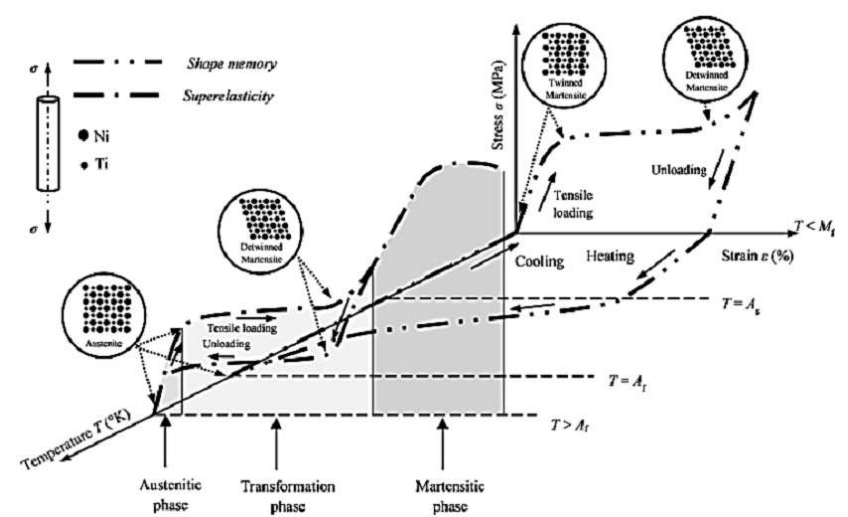

Fig. 2. Stress-strain-temperature diagram exhibiting the shape memory and superelastic effect of $\mathrm{NiTi}$ alloy. Crystal transformation is caused due to changes in temperature and stress [5].

Because of this functional mechanical behaviour ( $\mathrm{su}-$ perelasticity) NiTi wires were used in the first (initial) phase of orthodontic treatment [6], which deals with the alignment and derotation of teeth, as well as correction of vertical and horizontal discrepancies by levelling the dental arches. In order to produce physiological response in the periodontal ligament (PDL) and bone the initial aligning wires should apply light continuous force on the teeth. The magnitude of the force caused by the orthodontic wire is largely dependent on the material properties (modulus of elasticity) [7].

The purpose of the present paper was to analyse the solidification and castings process of a Ni-Ti binary alloy with general composition 50.68051 at.\% Ni and 49.31949 at.\% $\mathrm{Ti}$ (or in wt.\% $55.749516 \mathrm{Ni}$ and $44.250483 \mathrm{Ti}$ ). In the first step we defined the chemical composition according to the curve in Fig. 3.

From the measured values a polynomial equation was calculated in which the dependence of the $A_{\mathrm{f}}$ temperature from the Ni content is shown

$$
y=-114 x^{2}+11385 x-284161 .
$$

From the condition that the $A_{\mathrm{f}}$ must be about $26^{\circ} \mathrm{C}$ which is lower than the body temperature $\left(36.5^{\circ} \mathrm{C}\right)$, we defined the appropriate content of $\mathrm{Ni}$ and, consequently, the whole composition of the NiTi alloy (see Table I).

This study investigated the possibility of production simplicity by melting in further continuous vertical casting. For this purpose in the first step we used solidification and casting processes modelling with the numerical approach.

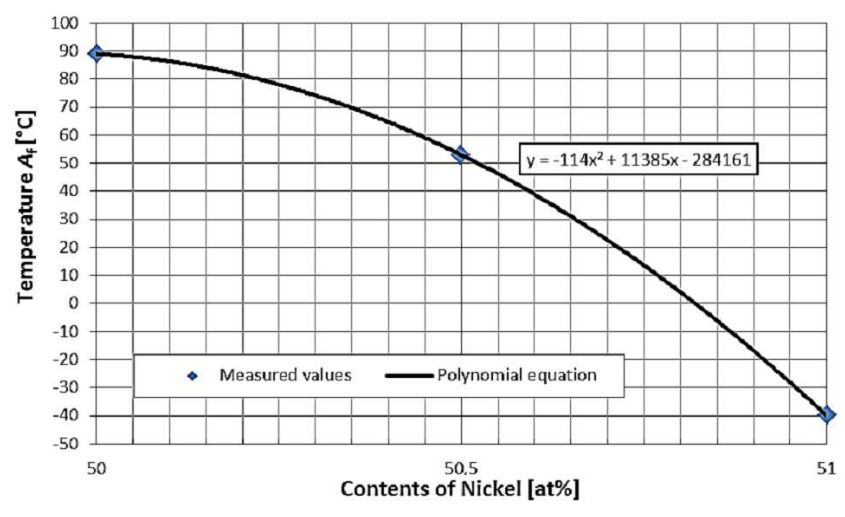

Fig. 3. Curve of temperature $A_{\mathrm{f}}$ dependence from Ni contents (measured values, polynomial equation).

TABLE I

$A_{\mathrm{f}}$ temperature in dependence of Ni content.

\begin{tabular}{c|c|c}
\hline \hline$A_{\mathrm{f}}\left[{ }^{\circ} \mathrm{C}\right]$ & $c$ & $\mathrm{Ni}[\mathrm{at} \%]$ \\
\hline 28 & 284189 & 50.66866 \\
27 & 284188 & 50.67461 \\
26 & 284187 & 50.68051 \\
25 & 284186 & 50.68636 \\
24 & 284185 & 50.69217
\end{tabular}

\section{Numerical method}

\subsection{Governing equations}

The time-dependent NiTi alloy solidification using ANSYS Fluent was modelled by the enthalpy-porosity technique [8]. In this technique the melt interface is not tracked explicitly. Instead, a liquid fraction, which indicates the fraction of the cell volume that is in liquid form, is associated with each cell in the computational domain.

The liquid-solid mushy zone is a region in which the liquid fraction values lie between 0 and 1 and its temperature ranges between the liquidus $\left(T_{1}\right)$ and solidus $\left(T_{\mathrm{S}}\right)$ temperatures. The mushy zone is modelled as a "pseudo" porous medium in which the porosity decreases from 1 to 0 as the material solidifies. When the material has solidified fully the porosity becomes 0 and hence the velocities also drop to 0 .

The enthalpy of the material is computed as the sum of the sensible enthalpy $(h)$ and the latent heat $(\Delta H)$ :

$$
H=h+\Delta H=h_{\text {ref }}+\int_{T_{\text {ref }}}^{T} c_{p} \mathrm{~d} T+f_{1} L_{v},
$$

where $h_{\text {ref }}$ is reference enthalpy, $T_{\text {ref }}$ - reference temperature, $c_{p}$ - specific heat at constant pressure, $f_{1}$ liquid mass fraction and $L_{v}$ - latent heat of fusion.

The liquid fraction, $f_{1}$, is defined as:

$$
f_{\mathrm{l}}=\left\{\begin{array}{cc}
1 & T>T_{1} \\
\left(T-T_{\mathrm{s}}\right) /\left(T-T_{\mathrm{s}}\right) & T_{\mathrm{s}}<T<T_{1} \\
0 & T<T_{\mathrm{s}}
\end{array}\right.
$$

Finally, for the solidification problem, the energy equation reads as 


$$
\frac{\partial}{\partial t}(\rho H)+\nabla \cdot(\rho \boldsymbol{v} H)=\nabla \cdot(k \nabla T),
$$

where $H$ is enthalpy (see Eq. (1)), $\rho$ is density and $\boldsymbol{v}$ is velocity.

The conservation equation of mass and momentum are decoupled from the one of the thermal energy. These equations are solved using a segregated solver with the second order accurate upwind scheme.

\subsection{Geometry and boundary conditions}

The problem of the NiTi alloy solidification in the experimental tube of length $L$ and diameter $D$ is depicted schematically in Fig. 4.
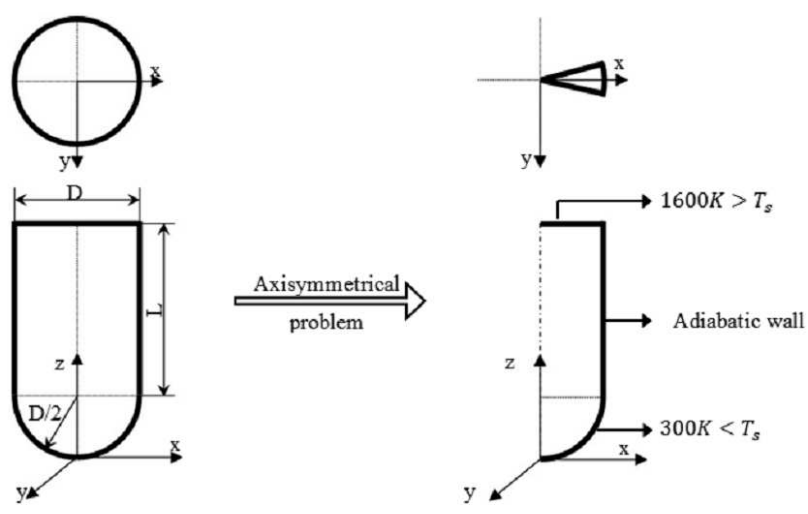

Fig. 4. Schematic representation of geometry.

Due to the symmetrical geometry, the NiTi alloy solidification was modelled as an axisymmetrical problem. The upper horizontal and lower curved walls were maintained at the constant temperatures, whereas the other boundaries are considered to be adiabatic in nature. The temperature of the curved wall was lower than the solidus temperature in order to allow the solidification and provide the phase change. The physical properties of the melt are given in Table II.

\section{TABLE II}

Thermal properties used in the analysis.

\begin{tabular}{l|l}
\hline \hline Density & $\begin{array}{l}\text { Variable } \\
\text { (from experiments) }\end{array}$ \\
\hline specific heat & $0.7988 \mathrm{~J} /(\mathrm{kg} \mathrm{K})$ \\
latent heat & $24200 \mathrm{~J} / \mathrm{kg}$ \\
thermal conductivity & $21.5 \mathrm{~W} /(\mathrm{m} \mathrm{K})$ \\
viscosity & $0.00574 \mathrm{~Pa} \mathrm{~s}$ \\
solidus temperature & $750 \mathrm{~K}$ \\
liquidus temperature & $1310 \mathrm{~K}$
\end{tabular}

Such a geometrical setup (adiabatic wall) will allow us to perform further directional solidification experiments, in which the solidification processing parameters (e.g. temperature gradient and growth rate) can be controlled independently [9] so that one may study the dependence of the microstructural parameter on either temperature gradient at constant growth rate or growth rate at constant temperature gradient for the constant initial NiTi solute composition.

\section{Grid refinement and numerical accuracy assessment}

The grid independence of the present results has been established on the basis of a detailed analysis using four different meshes (the elements were concentrated towards each solid wall). With each grid refinement the number of elements in a particular direction is doubled and the minimum element size is halved. Such a procedure is useful (and encountered in many numerical studies [1012]) for applying the Richardson extrapolation technique, which is a method for obtaining a higher-order estimate of the flow value (value at infinite grid) from a series of lower-order discrete values.

For a general variable $\phi$ the grid-converged value according to the Richardson extrapolation is given as

$$
\phi_{\mathrm{ext}}=\phi_{\mathrm{MIII}}-\left(\phi_{\mathrm{MIII}}-\phi_{\mathrm{MIV}}\right) /\left(r^{p}-1\right) \text {, }
$$

where $\phi_{\text {MIV }}$ is obtained on the finest grid and $\phi_{\text {MIII }}$ is the solution based on the next level coarse grid, $r$ is the ratio between coarse to fine grid spacing and $p=2$ is the expected order of accuracy.

The variation of liquid fraction and temperature with grid refinement is given in tabulated form in Table III. The "percent" numerical error as given in Table III is a quantification of the relative difference between the numerical predictions and the extrapolated value obtained with Richardson's extrapolation technique.

TABLE III

Effect of mesh refinement upon the liquid fraction and temperature at $(x=0$ and $z=0.1)$ for a steady-state numerical analysis.

\begin{tabular}{c|c|c|c|c|c|c}
\hline \hline & MI & MII & MIII & MIV & $\phi_{\text {ext }}$ & Error \\
\hline Num. el. & 4700 & 18800 & 75200 & 300800 & $/$ & $/$ \\
$f_{1}$ & 0.5410 & 0.5401 & 0.5396 & 0.5393 & 0.5391 & $0.06 \%$ \\
$T$ & 1052.95 & 1052.43 & 1052.17 & 1052.03 & 1051.90 & $0.02 \%$
\end{tabular}

The results of calculations of numerical accuracy indicate that, as the mesh is refined, there is a consistent improvement in the accuracy of the predicted values, and the agreement between predictions obtained with mesh MIII and the extrapolated value is extremely good. Based on this, the simulations in the remainder of the paper were conducted on mesh MIII, which provided a reasonable compromise between high accuracy and computational effort.

\section{Results and discussion}

\subsection{Flow field}

The temperature gradients and resulting density variation induce the natural convection. As shown in Fig. 5, the movement due to the natural convection is rather slow and reduces as the time progresses. Due to that, 
one can conclude that, in the case of NiTi solidification in an experimental tube, the heat conduction is the sole transport mechanism.

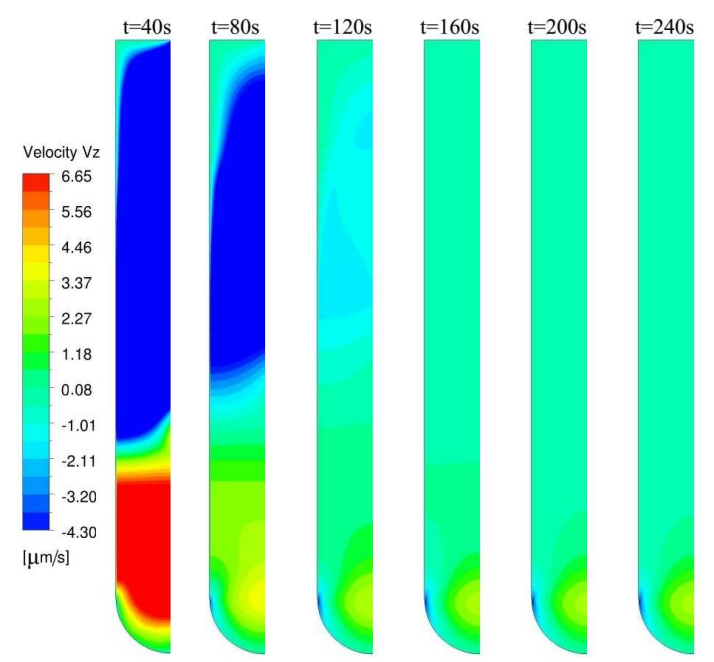

Fig. 5. Contours of vertical velocity.

\subsection{Temperature field}

Figure 6 presents the temperature field evolution with the time. The temperature contours are mainly lines, which signify that the heat transfer takes place by the conduction mechanism. The exception is the region in the vicinity of the lower curved wall, where contours of the temperature are slightly curved. However, even in this region, the heat conduction is the prevailing mechanism and the curved contours result from the curved geometry of the lower wall.

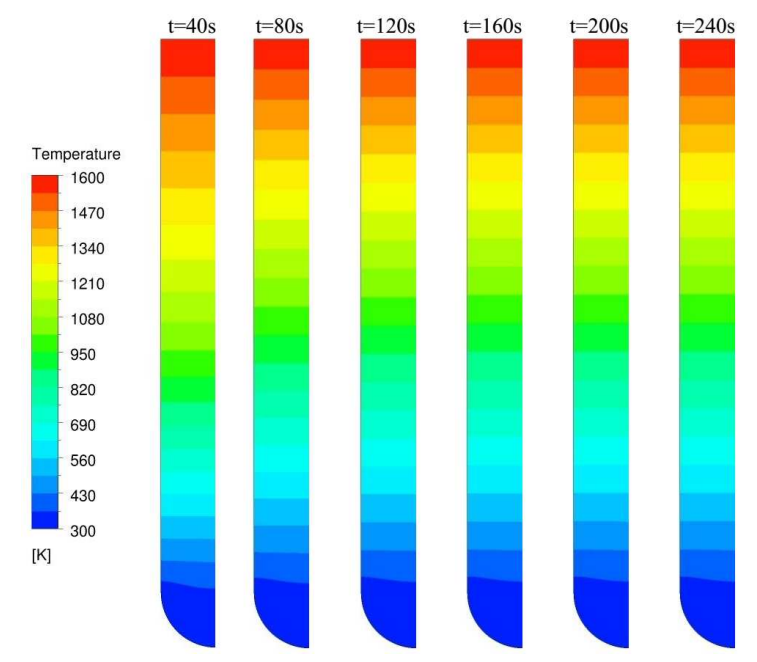

Fig. 6. Contours of temperature.

\subsection{Solidification/liquid mass fraction}

Figure 7 presents the contours of NiTi liquid mass fraction during the solidification. Contours are lines signifying the unidirectional solidification process in the experimental tube. Furthermore, the solidification front moves upward, which is consistent with the time evolution of the temperature field.

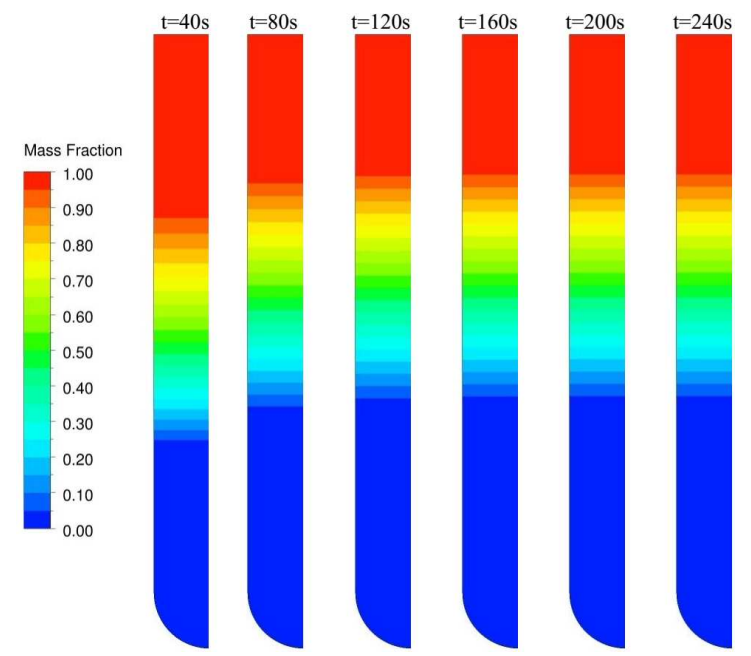

Fig. 7. Contours of NiTi liquid mass fraction.

\section{Conclusions}

In the present study the time-dependent phase-change of NiTi alloy in an experimental tube was studied by numerical means. The solidification process was modelled by the enthalpy-porosity method.

The influence of computational grid refinement on the present numerical predictions was studied throughout the examination of grid convergence. By utilising extremely fine meshes the resulting discretisation error levels are below $0.10 \%$.

Highly accurate numerical results pointed out some important points, such as:

- The calculated flow field shows slight movement in the vertical direction, and reduces as the solidification time progresses.

- The temperature is transported mainly by the conductive heat transfer mechanism.

- The solidification process in an experimental tube is unidirectional.

- The solidification front moves upward.

Last but not least, the present numerical approach and results will be compared with the experimental one which will give us enough confidence to continue with the numerical analysis of NiTi alloy continuous casting process on the lab-scale.

\section{Acknowledgments}

The research leading to these results was carried out within the framework of the Research Project "Biomedical shape memory alloys" (L2-5486) and has received funding from the Slovenian Research Agency (ARRS). 


\section{References}

[1] D. Cimprič, in: Shape Memory Alloys, Seminary Work, Faculty of Mathematics and Physics, Department of Physics, Ljubljana 2007.

[2] M. Thier, D. Treppmann, D. Drescher, C. Boureaul, J. Mater. Sci. Mater. Med. 3, 229 (2004).

[3] B. Coluzzi, A. Biscarini, L. Di Masso, F.M. Mazzolai, N. Staffolani, M. Guerra, M. Santoro, S. Ceresar, A. Tuissi, J. Alloys Comp. 233, 197 (1996).

[4] K. Otsuka, X. Ren, Prog. Mater. Sci. 50, 511 (2005).

[5] J. Ferčec, M. Kos, M. Brunčko, I. Anžel, B. Glišić, E. Marković, R. Rudolf, Materiali in tehnologije 48, 99 (2014).

[6] J. Ferčec, I. Anžel, R. Rudolf, Mater. Des. 55, 699 (2014).
[7] J. Ferčec, B. Glišić, I. Šćepan, E. Marković, D. Stamenković, I. Anžel, J. Flašker, R. Rudolf, Acta Phys. Pol. A 122, 659 (2012)

[8] V.R. Voller, C. Prakash, Int. J. Heat Mass Transf. 30, 1709 (1987).

[9] M. Gündüz, H. Kaya, E. Çadırlı, N. Maraşlı, K. Keşlioğlu, B. Saatçi, J. Alloys Comp. 439, 114 (2007).

[10] I. Biluš, M. Morgut, E. Nobile, Int. J. Simul. Model. 12, 94 (2013).

[11] P. Ternik, R. Rudolf, Int. J. Simul. Model. 12, 5 (2013).

[12] P. Ternik, R. Rudolf, Int. J. Simul. Model. 13, 312 (2014). 